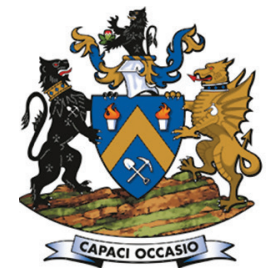

Affiliation:

1 Department of Civil,

Environment and Natural

Resources Engineering Division

of Mining and Rock Engineering

Luleå University of Technology,

Luleå, Sweden.

2 Division of Decision and Control

Systems, School of Electrical

Engineering and Computer

Science, KTH Royal Institute

of Technology, Stockholm,

Sweden.

${ }^{3}$ ABB Corporate Research

Center, Västerås, Sweden.

4 SimMine, Malå, Sweden.

${ }^{5}$ Department of Chemical and

Mining, College of Engineering and Technology, The University of Dar es Salaam, Tanzania.

${ }^{6} \mathrm{ABB}$ Industrial Automation,

Process Industries, Umeå,

Sweden.

Correspondence to:

B. Skawina

Email:

bart.skawina@ltu.se

Dates:

Received: 20 Mar. 2020

Revised: 11 Sep. 2020

Accepted: 10 Mar. 2021

Published: June 2021

\section{How to cite:}

Skawina, B., Astrand, M.,

Sundqvist, F., Greberg, J.,

Salama, A., and Ekbeck, P. 2021

Automatic closed-loop scheduling

in underground mining using

discrete event simulation.

Journal of the Southern African

Institute of Mining and Metallurgy,

vol. 121, no. 6, pp. 277-282.

DOI ID:

http://dx.doi.org/10.17159/2411-

9717/679/2021

ORCID

B. Skawina

https://orchid.org/0000-0002-

7334-4934

M. Astrand

https://orchid.org/0000-0001-

6778-51544

J. Greberg

https://orchid.org/0000-0002-

2306-9241

\title{
Automatic closed-loop scheduling in underground mining using discrete event simulation
}

\author{
by B. Skawina1, M. Astrand ${ }^{2,3}$, F. Sundqvist'1,4, J. Greberg1, \\ A. Salama ${ }^{5}$, and P. Ekbeck ${ }^{6}$
}

\begin{abstract}
Synopsis
Today's mining operations require fast reporting and rapid rescheduling based on updated information. An automatic mine scheduling system could not only quickly reschedule but also propose alternative solutions. To avoid the financial and physical risks associated with testing such a system directly in operation, it could be first evaluated via discrete event simulation models. This would offer a safe environment to evaluate different operating rules and algorithms. In this study, this is achieved by integrating automatic scheduling software with a discrete event simulation model.
\end{abstract}

\section{Keywords}

discrete event simulation; production planning; scheduling system; underground mining.

\section{Introduction}

Traditionally, production planning in mining operations has been divided into four levels (Figure 1). In long-term planning, the focus is on formulating rough plans for mine production over a long period. These plans change over time to reflect the effects of a changing economy and market demand for minerals. Long-term plans can be used to establish financial forecasts relevant for the purchase of new equipment, changes in the workforce, and variations in operating costs (Hartmann and Mutmansky 2002). Long-term planning allows mine planners to first set mining targets and then test scenarios to find the best way to achieve the targets over multiple working periods. Medium-term and short-term plans contain a level of detail that reflects the immediate needs of the mine. These plans provide the information necessary for future production forecasts and predictions of market demands; they usually include daily production rates, material handling plans, equipment, and resource allocation. Production scheduling creates detailed schedules for a short period to ensure control of the ore grade, consistent daily operations (following a monthly plan), effective use of available resources, and maximum utilization of resources within the operating constraints (Hartmann and Mutmansky 2002).

Unexpected events can cause disturbances in operations, creating problems that a production planner must solve daily. In underground mining operations, manual rescheduling is time-consuming and production planners often find themselves in a situation where new or readjusted schedules are required in a very short time. It is also difficult to maintain a high quality of reporting and may even lead to a loss of important information.

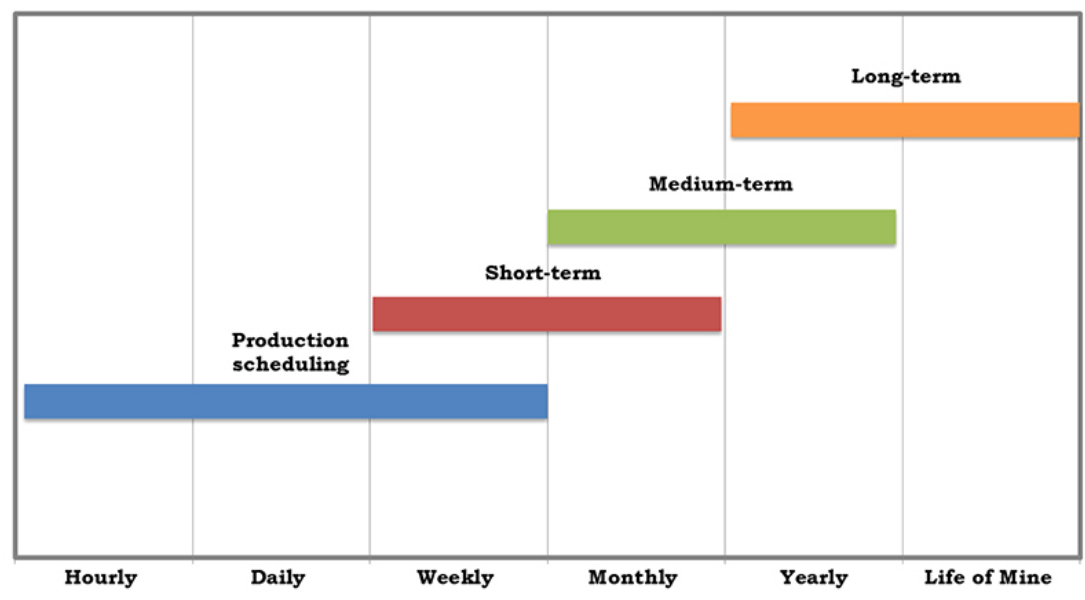

Figure 1-Time horizons for production plans in mining 


\section{Automatic closed-loop scheduling in underground mining using discrete event simulation}

An automated scheduling system, with real-time production feedback, could help planners initiate the necessary changes within a short time when unexpected events occur or when task execution can be improved. To avoid the financial and/ or physical risks of testing the system in a real environment, automatic closed-loop scheduling can be connected to a simulation system. Tests in the real mine will still be required, but with simulation, many early issues may be resolved before changes are implemented in the mine operations. The challenge is threefold: to evaluate and accommodate complex and changing restrictions, to generate optimized production plans that meet production targets, and to promptly activate new production plans when unexpected errors occur. Introducing such an approach will help to optimize short-term production scheduling, reduce overall production costs and time, increase equipment efficiency, solve complex scheduling problems, and improve decision-making by increasing the efficiency and agility of planning tasks that are subject to change. This, in turn, will reduce operator stress, provide instant task progress reports, reduce the administrative workload, coordinate mine systems with fewer human errors, and unify scheduling.

This paper discusses the automatic scheduling tool and the commercial discrete-event simulator to create a possible digital twin that can be used to test various mine scenarios. This study was part of the Optimized Medium-Range Mine Scheduling (OMMS) project and was carried out by Luleå University of Technology (LTU), ABB, Boliden, and SimMine, coordinated by RockTech Centre, and funded by the partners and SIP STRIM. Further studies have been a part of the Sustainable Intelligent Mining Systems (SIMS) project and the H2020 EU Research and Innovation Programme.

\section{Scheduling and simulation}

Scheduling is defined by Pinedo (2002), Brucker (2004), and Dugardin et al. (2007) as a process of allocating resources (people, equipment, or production lines) to tasks/jobs over time. The goal is to optimize (maximize or minimize) certain objectives, such as processing times or quantities, by making appropriate decisions (Dugardin et al. . 2007; Fowler et al. . 2006). When assigning the resources, decisions must be based on the production targets and other factors, such as priorities, sequencing, beginning and end of the job/task, but also including unexpected stops and rescheduling (Herrmann 2006). With the increasing complexity of systems and changing restrictions, scheduling should undergo a continuous cycle of improvement. However, the manual process of generating schedules consumes a lot of time, is prone to errors, and makes it difficult to incorporate real-time information. Thus, while production planners currently use their own experience and personal judgment (Song et al. 2015) in scheduling, they could be assisted by automated production scheduling systems.

Discrete event simulation (DES) is a widely used and suitable method for analysing dynamic systems in mining. In DES, variables change at discrete points when events occur (Banks 1998). An event can be an arrival or departure of a machine, for example. Simulation models are typically used for tactical and strategic decision support to solve problems that cannot be tackled by analytical techniques (Fowler and Rose 2004). They offer a solution to a problem in a controlled environment. One of the key advantages of simulation is that it does not disturb the existing system (Dugardin et al.., 2007).
There are various options for simulation and scheduling. The first option involves using the simulation as a base schedule for generating the initial schedules, improving the existing schedules, or optimizing the existing schedules from within the simulation (Fowler, Mönch, and Rose, 2006). This means the simulation model is a production control system used to derive production control instructions (Fowler, Mönch, and Rose, 2006). In an example given by Potradi, Maso, and Fowler (2002), a DES model was used to generate the schedule for machines and to plan the start of operations. A second option for simulation is to set parameters or test the instance generation required for scheduling. In this case, simulation is used to evaluate certain parameter setting strategies for a given production control scheme (Fowler, Mönch, and Rose, 2006). A third option is to use simulation to emulate and evaluate scheduling approaches. This allows the behaviour of a system to be evaluated. However, the simulation software has to have the functionality to model specific characteristics of the system (Mönch, Rose, and Sturm, 2003). Horiguchi et al. . (2001) and Pritsker, O'Reilly, and Laval (1997) examined the performance of the algorithm by developing a simulation model in the AweSim DES language, while Mönch Rose, and Sturm (2003) evaluated scheduling approaches in a simulation-based environment for manufacturing systems.

In this study, a connection between an automatic scheduler and a discrete event simulation (DES) tool is established to create a possible digital twin of the mine that could be used in the future to test various scenarios. This study considers the use of DES to emulate and evaluate the scheduler, with a focus on system interaction, scale of operation, and interaction between the underground mine model and the automatic production scheduling system. The simulator and scheduler thus jointly act as a test bed and decision support system. This enables the user to evaluate the automatic scheduling, current operating scheduling rules, and potential improvement strategies in closeto-real mine conditions without disturbing production.

\section{Integration of automatic scheduling system and DES model}

The following section describes the integration of an automatic scheduling tool, the ABB Ability Operations Management System Execution Manager (OMS EM), and a DES tool, SimMine. The focus is on closing the loop in short-term mine scheduling by using simulation as a test bed and decision support system. OMS EM is a system for managing processes through detailed production schedules based on information collected from various sources. The system supports manual scheduling and intervention to enable such techniques as short interval control. An additional feature of OMS EM is the possibility to automatically create short-term schedules using algorithms. Scheduling is typically considered a manufacturing execution system, the intermediate layer between enterprise resource planning and production control. This means any industrial application of a manufacturing execution system must be tightly integrated with the (possibly complex) IT system on site. With this in mind, OMS EM was developed to support processes that can be modelled using the ISA-95 standard (ANSI/ISA-95.00.03 2005). The adherence to ISA-95 makes the integration with various information sources easier, as a common data model is used. Furthermore, in the mining industry, the standard is recognized by many companies as an enabler for future operation optimization (Roberts, 2016). Lastly, the automatic 


\section{Automatic closed-loop scheduling in underground mining using discrete event simulation}

scheduling feature in OMS EM enables closed-loop scheduling by incorporating real-time task progress from telemetry or mobile clients used by the production. Automatic scheduling is thus used to continuously reschedule based on the latest information available. Information is presented in the form of Gantt charts in which information is continuously updated (see Figure 2). In this study, machine location, machine activity, machine alarms, and delays are shown. Users can interact (in the Gantt chart) with the current automatically created schedule by reassigning orders to different equipment or editing the properties of the resources.

SimMine is a mining-oriented DES tool built on a full graphical interface. Since SimMine was developed specifically to simulate mining operations, it is made of interfaces where the user can set and specify underground mine-related parameters, such as fleet type and properties, rock properties, location sequences, shifts, and processing times. SimMine software accommodates various mine layouts and produces comprehensive output statistics. In this work, we develop a number of new modules to represent the surrounding information system, including extraction plans and maintenance systems. Based on the information obtained from the studied mine, SimMine can not only simulate the process, but also act as the mine operation centre (MOC) to coordinate activities.

The SimMine model of the simulated mine is shown in Figure 3. The figure includes the main interface and the graphical user interface (GUI). The main interface is split into main menu toolbars and tabs. Each tab consists of different input/output data for the current simulation. Input data includes fleet data (shift properties, activity cycles, fleet properties, operator costs, and materials), and layout data (face profiles, location type, dependencies, priorities, rock properties, and capacities). Output data includes the statistics related to sequence, development, production, location, fleet performance measures, activity and loading, and transport. The GUI represents the layout of the 3D modelled scenario; it includes the tools and objects used for selecting, creating, or modifying the layout and views two perspectives: machine-centric and location-centric.

\section{Platform integration}

The OMS EM scheduling system is responsible for generating schedules and assigning resources (or equipment) to tasks. The SimMine DES simulation is responsible for simulating the real mining system and reporting events to the scheduler (Figure 4). The layout of SimMine and OMS EM integration includes information related to the production/development plan, maintenance plan, system settings, localization, and additional mine services that can affect the scheduling in the mine.

At the start of the simulation, the simulator sends the location dependencies (available faces), order list (blast orders), and tasks (full set of drill and blast activities that should be performed in a particular set-up) to the scheduler (Figure 5).

During the simulation, vacant vehicles request work from the scheduler. Work comes in the form of work orders. When machines perform work, their work status is transferred to the scheduler (start/stop of activities and expected end time). To communicate and exchange information, OMS EM and SimMine use web services. The information is presented to users in the form of a Gantt chart (Figure 2). In the chart, activities such as the current status of work, finished or future mining activities are grouped depending on the orders, type of equipment, type of material, and location. This improves the decision-making for the automatic scheduling of the production cycle by sending

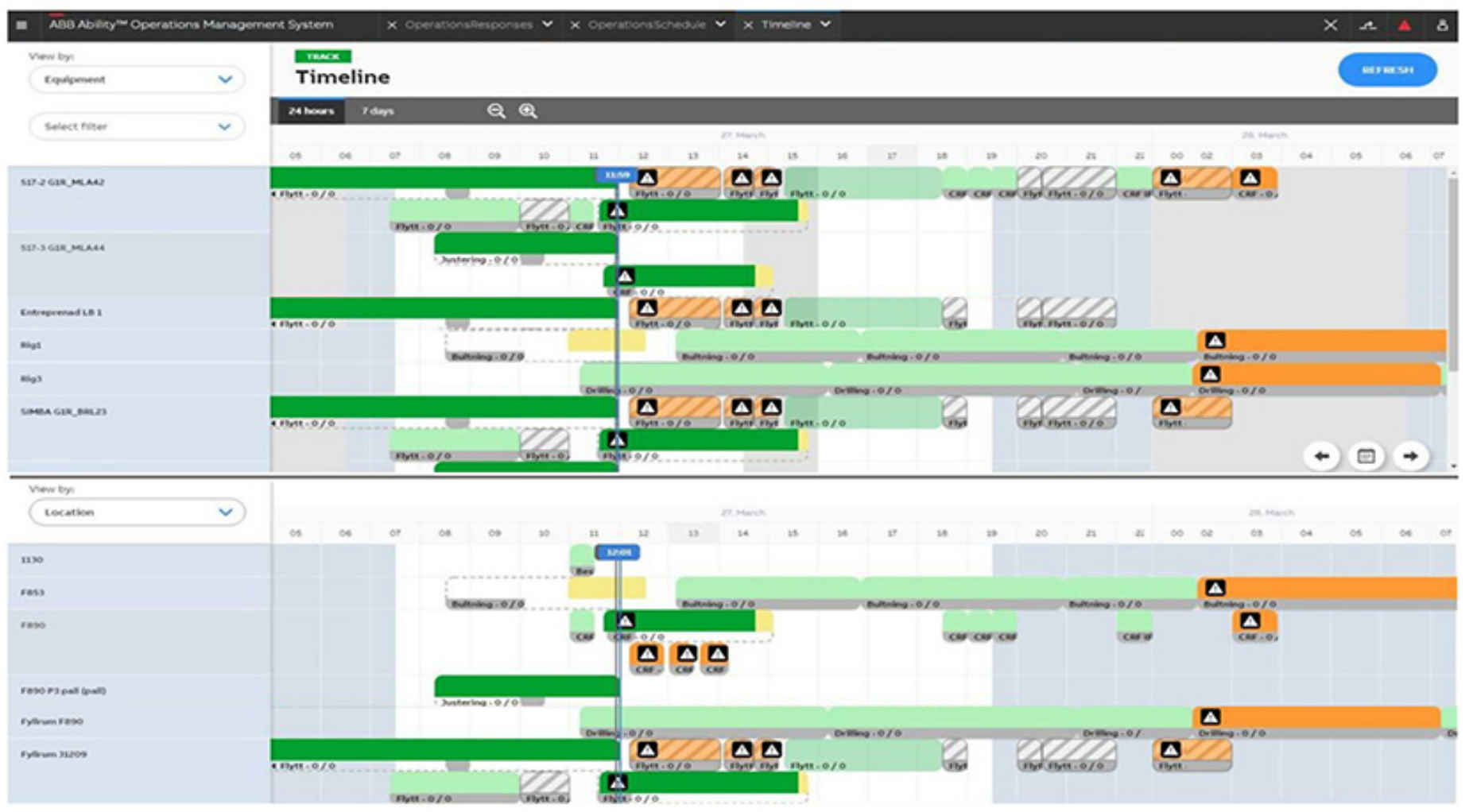

Figure 2-Views of the scheduling system. The top diagram presents a machine-centric Gantt chart; the bottom diagram presents the same information from a location-centric view 
Automatic closed-loop scheduling in underground mining using discrete event simulation

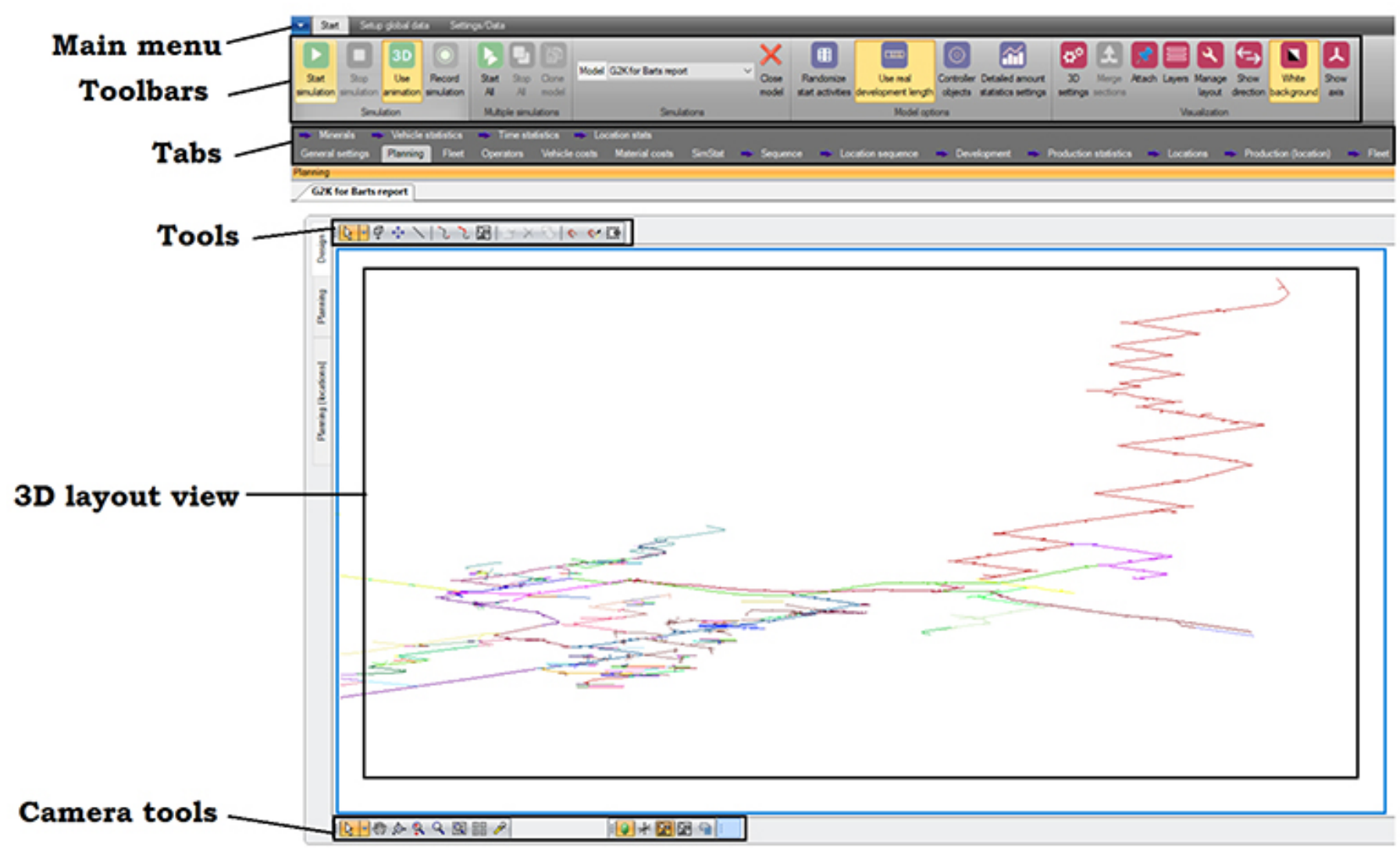

Figure 3-Simulation model

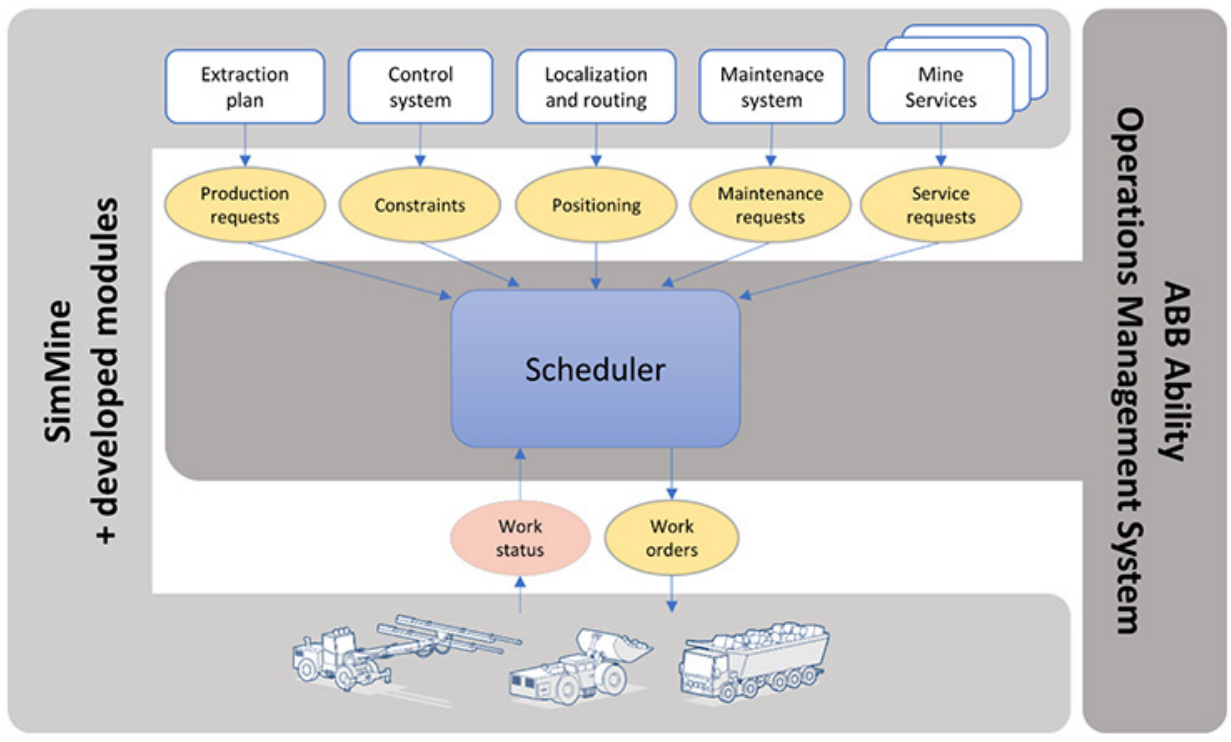

Figure 4-Layout of the integration of the scheduler and the simulator

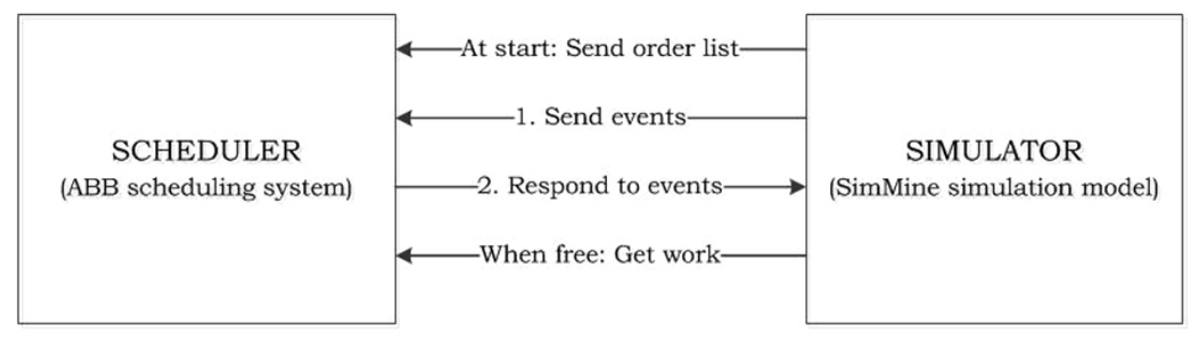

Figure 5-Platform interaction 


\section{Automatic closed-loop scheduling in underground mining using discrete event simulation}

feedback on work progress, together with reasons for machine interruptions, from each operation to the fleet management system.

\section{Simulation results}

Multiple simulation runs are performed to check the functionality and quality of both the simulator and the scheduler. The main objective in this example is to demonstrate the joint system, and evaluate whether the automatic scheduler can achieve the same production results as schedules based on operational data. The designed system was tested on a small-scale operation (limited set of faces). The results are an example of two 2-week simulations runs.

The results presented in Figure 6 show the cumulated developed distance for both the base case scenario, using operational data with added activity switch time and working rules, and the scenario when using automatic scheduling. The activity switch time is a time penalty of around 20 minutes after a vehicle has finished a task until it can continue with the next task. The activity switch time is simulated to represent the reallife scenario where the operator is waiting to receive the next work order from the mine control centre. The work rules are the rules which prohibit the vehicle to start work at a face if there is 15 minutes or less time until a break occurs (shift ends or meal break). The developed distances achieved for the base case and the scenario when using automatic scheduling were $210 \mathrm{~m}$ and $217 \mathrm{~m}$, respectively. For these two weeks, the comparison shows that the scenario where automatic scheduling was used produced similar results.
The results presented in Figures 7 and 8 show the machine utilization for the base case scenario and the scenario using automatic scheduling. Machine utilization is the effect of performing the work, where the work itself, shows similar results between the cases. Based on the comparison of the results between Figure 7 and Figure 8, the difference in the work time is the highest for three machines: bolter 1 (10\%), drill rig, 3 (18\%), and loader $(11 \%)$. The results also show that the difference in the idle time is the highest for loader (24\%), LHD 1 (20\%), scaler 1 (18\%), drill rig $3(18 \%)$, and bolter $1(18 \%)$. A comparison of the utilization of the machines shows that in the case of the scheduler the utilization is slightly lower compared with the base case scenario. Since the production results are almost matching, the difference in machine utilization originates from different guiding operating rules in the scheduling process.

\section{Concluding remarks}

This study integrates an automatic scheduling system with a DES model. The joint system can achieve the same production results as schedules based on operational data. The simulator supports most of the functionality required to carry out a performance assessment, with all the required details. The proposed system can act as a test bed and a decision support system for functionality checks and quality controls. This enables the user to evaluate the schedules produced by the scheduling platform without interacting with existing systems or processes. When the scheduler is connected to the simulator, the mine operation centre can continue to update and use the simulator to test different algorithms and procedures/rules applied to the schedule or to

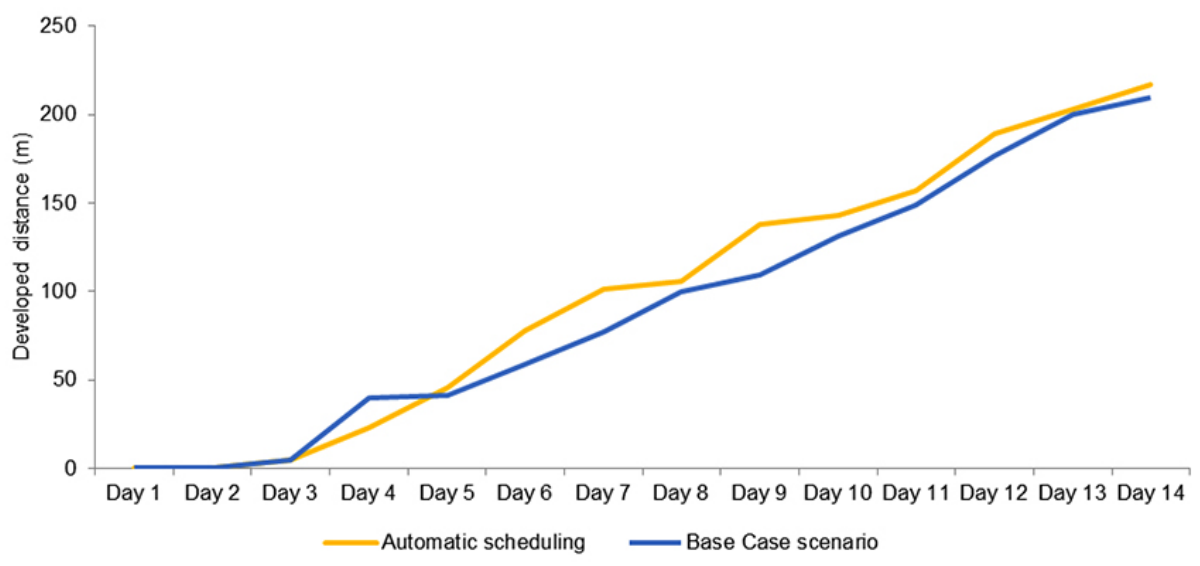

Figure 6-Cumulated developed distance

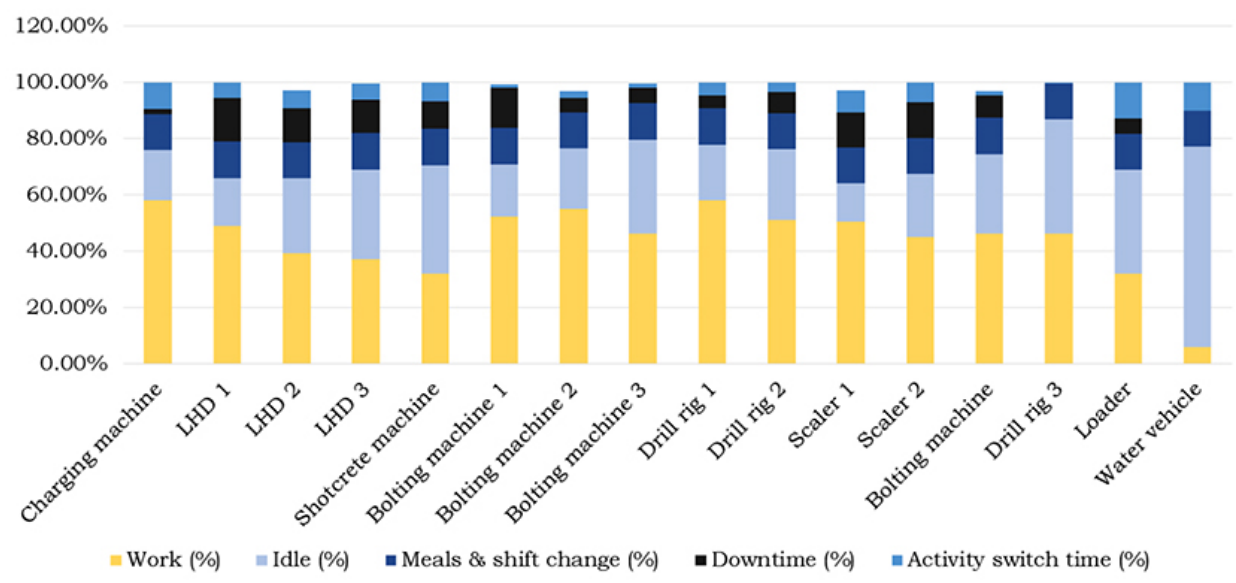

Figure 7-Base case scenario 


\section{Automatic closed-loop scheduling in underground mining using discrete event simulation}

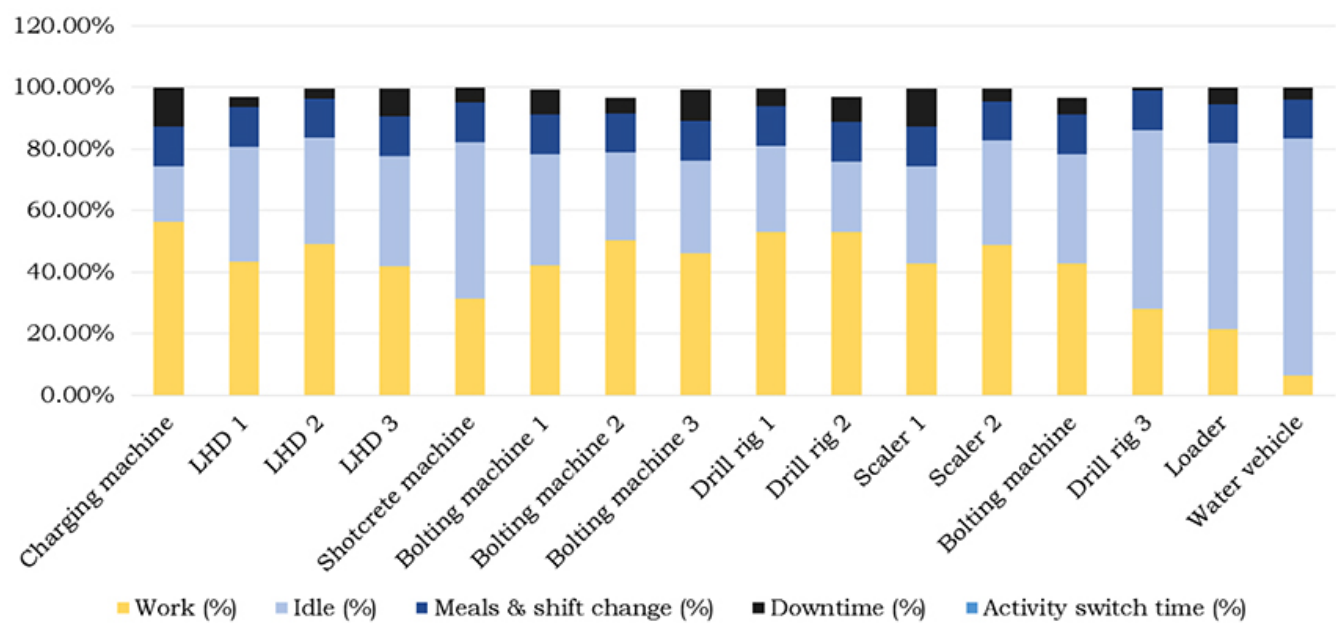

Figure 8-Scenario when using automatic scheduling

see the results of applying a specified production schedule in the simulator beforehand. In this way, the scheduling process can be tested in situations that could occur in a real environment, rather than in simple predefined scenarios. This opens the possibility to examine the impact of automatic scheduling compared to the current situations in mines, where production scheduling is highly manual.

\section{Future development}

Formulating mathematical models to represent machine environments is very complex and subject to complex policies and rules related to sequence-dependent set-ups (Sivakumar 2001). The level of detail is an important part of the design, as it may increase the credibility of the model but also increase the complexity and execution time (Fowler and Rose 2004). The scheduler's primary function should be to reduce the time and effort necessary to produce feasible production schedules and to guide production management in the decision-making process. Therefore, future factors to consider when increasing the level of detail are rock mechanics, geological mapping, media, preventive maintenance, and material buffer capacity in different locations. An ongoing challenge in this project is to create the synchronized model representations in both the scheduler and the simulator. A future consideration is to leverage a standardization protocol for the automatic synchronization of these models.

\section{References}

AlbReChT, M.C. 2010. Introduction to DES. http://www.albrechts.com/mike/DES/ Introduction\%20to\%20DES.pdf [accessed 20 May 2017].

ANSI/ISA-95.00.03-2005. Enterprise-control system integration. Part 3: Activity models of manufacturing operations management. http://www.mecatronica. eesc.usp.br/wiki/upload/8/83/ISA-95.00.03-2000_Draft.pdf [accessed 13 March 2018].

Banks, J., Carson, J.S., Nelson, B.L., and Nicol, D.M. 2010. Discrete Event System Simulation. Pearson Education, Hoboken, NJ.

BAnKs, J. 1998. Handbook of Simulation: Principles, Methodology, Advances, Applications, and Practice. Wiley, New York.

Biskup, D. and Feldmann, M. 2001. Benchmarks for scheduling on a single machine against restrictive and unrestrictive common due dates. Computers \& Operations Research, vol. 28. pp. 781-801.

BRUCKER, P. 2004. Scheduling Algorithms. 4th edn. Springer, Berlin.

CuDDIHY, C. 2017. GMSG: Open mining format to improve 3D interoperability. Global Mining Review. https://www.globalminingreview.com/specialreports/17112017/gmsg-open-mining-format-to-improve-3d-interoperability/ [accessed 6 March 2018].

Dugardin, F., Chehade, H., Amodeo, L., Yalaoui, F., and Prins, Ch. 2007. Hybrid job shop and parallel machine scheduling problems: Minimization of total tardiness criterion. Multiprocessor Scheduling: Theory and Applications. Levner, E. (ed.). Intech, Vienna. pp. 273-292.

Fowler, J.W., Mönch, L., and Rose, O. 2006. Scheduling and simulation. Handbook of Production Scheduling. Springer. Chapter 5, pp. 109-133.

FowLER, J.W. and Rose, 0. 2004. Grand challenges in modeling and simulation of complex manufacturing systems. Simulations, vol. 80, no. 9. pp. 469-476.

GonZALES, F.G. and DAVIS, W.J. 1998. Initializing online simulations from the state of a distributed system. Proceedings of the 1998 Winter Simulation Conference. Medeiros, E.F., Watson, E.F., Carson, J.S., and Manivannan, M.S., (eds). IEEE, Piscataway, NJ. pp. 507-513.

HALL, N.G. and PosNer, M.E. 2001. Generating experimental data for computational testing with machine scheduling applications. Operations Research. vol. 49. pp. 854-865.

Hartmann, H.L. and Mutmansky, J.M. 2002. Introductory Mining Engineering. 2nd edn. Wiley.

Herrmann, J.W. 2006. Decision-making systems in production scheduling. Handbook of Production Scheduling. Springer. Chapter 4, pp. 91-108.

Horiguchi, K., Raghavan, N., Uzsoy, R., and VenKateswaran, S. 2001. Finite-capacity production planning algorithms for a semiconductor wafer fabrication facility. International Journal of Production Research, vol. 39, no. 5. pp. 825-842.

Horn, R.L. 1971. Validation of simulation results. Management Science. vol. 17, no. 5. pp. 247-258.

Mönch, L., Rose, O., and Sturm, R. 2003. A simulation framework for the performance assessment of shop-floor control systems. Simulations. vol. 79, no. 3. https://doi.org/10.1177/0037549703256039

Pinedo, M.L. 2002. Scheduling: Theory, Algorithms, and Systems. 2nd edn. PrenticeHall, Englewood Cliffs, NJ.

Potradi, J., MAso, S., and Fowler, J.W. 2002. Using simulation-based scheduling to maximize demand fulfilment in a semiconductor assembly facility. Proceedings of the Winter Simulation Conference. IEEE, Piscataway, NJ. pp. 1857-1861.

PRITSKER, A.A.B., O'ReILly, J.J., and LAVAL, D.K. 1997. Simulation with Visual SLAM and AweSim. Wiley, New York.

Rippenhagen, C. and Krishnaswamy, S. 1998. Implementing the theory of constraints philosophy in highly reentrant systems. Proceedings of the 1998 Winter Simulation Conference. Medeiros, E.F., Watson, E.F., Carson, J.S., and Manivannan, M.S. (eds). IEEE, Piscataway, NJ. pp. 993-996.

RoBERTs, R. 2016. BHPB: Why ISA-95 is a game changer. Mining Magazine. https:// www.miningmagazine.com/innovation/news/1263186/isa-bhpbs-vision [accessed 1 February 2018].

SCHLEsinger, P. 1974. Developing standard procedures for simulation validation and verification. Proceedings of the Summer Computing Simulation Conference, vol. 1. Society for Computer Simulation, San Diego, CA. p. 927.

SivaKumar, A.I. 2001. Multiobjective dynamic scheduling using discrete event simulation. International Journal of Computer Integrated Manufacturing, vol. 14 , no. 2. pp. 154-167.

Song, Z., Schunnesson, H., Rinne, M., and Sturgul, J. 2015. Intelligent scheduling for underground mobile mining equipment. PLOS One. https://journals.plos.org/ plosone/article?id=10.1371/journal.pone.0131003 [accessed 3 January 2018].

YUCESAN, E. and FowLER, J. 2000. Simulation analysis of manufacturing and logistics systems. Encyclopedia of Production and Manufacturing Management, Kluwer Academic, Boston, MA. pp. 687-697. 